\title{
A community officer's perspective of a rural hospital in KwaZulu-Natal
}

Amidst the seeming desolation here, I feel inspired each morning when I wake up to the sound of the cock crowing, and the morning star twinkling above the mountain backdrop. This feeling has been matched only by the sense of opportunity I perceive at the hospital every day. There might be a lot more hospital now than some 20 years ago, but there is still so much that can and must be done here. It is not surprising that the revolutionary work of Sydney Kark, and many others, emanated from their experiences in this very setting. Necessity is, after all, the mother of invention.

I have spent the past few weeks appreciating the complexities of my new world. A real sense of the true Ubuntu spirit, juxtaposed with the tragedy of the limitless and depredatory nature of poverty. I am fortunate to be working in what is essentially a communicable diseases (TB/HIV) ward, and I am startled by the growing problems of treatment interruptions, delayed presentations, and the strong desire by many to assume the sick role right alongside the most stoic of individuals. The opportunity to learn from these patients has never been more obvious. The tangible impact of social determinants of individual, community and public health has never seemed clearer. Some concepts, like 'access' to healthcare, simply cannot be appreciated from afar - not with the same depth of appreciation, at least. Together with a resignedly ageing population who have largely given up on the hope of a better, brighter tomorrow, is a growing, young and restless population who are becoming increasingly tired of waiting for the tide to turn. Both groups cope with their frustrations in their own way. What is left is a desperate reliance on the 'disability' grant as a financial crutch, the consumption of whole families by treatable conditions such as TB and HIV, shocking levels of alcoholism and its associated violence, and the leaden feeling in your stomach when you realise that the Gini co-efficient isn't just a concept in economics textbooks. I have great difficulty identifying ways of making healthcare more 'accessible' to our community, and trying to understand how to build a greater local awareness of the inextricable link between poverty, disease and health-seeking behaviour. I have tried to understand the huge burden that TB places on individuals in this community, apart from the obvious clinical impact. The cost of presenting for daily streptomycin injections, for example, appears to top the list for many treatment interrupters. Social stressors, family responsibility, and the lack of food security seem to follow close behind. I've spent many hours pondering the more obvious challenges, too - poor infection control, lack of patient education, the disjunction between TB and HIV services, and little understanding of the local disease epidemiology.

From a much broader perspective, I have also been taken aback by the unique and refreshingly unfamiliar way in which 'small' district hospitals run - filled with many contrasts to busy, mechanistic, impersonal, urban hospitals. Healthcare workers in general, and doctors in particular, seem much happier here. Perhaps this is because
I am in a happier space in my own life compared with overwhelming internship, but my interactions with the doctors who work here tells me that they are genuinely more content. This is probably thanks to several factors, but my suspicion is that it is directly related to the palpable difference in organisational culture and the benefits of working in smaller, close-knit teams. The enthusiastic nursing staff and a greater sense of being needed and valued are rewarding for many doctors here. This spirit is something that centralised healthcare is desperately lacking - a culture of teamwork, co-dependence, acknowledgement of value, appreciation and reward.

The flipside of this small-town functionality, however, is the slow and sometimes non-existent progress towards improvement. Complacency is a threat to the survival of district level healthcare. The lack of formal management and operational training of most managers here means that there is no scientific, evidenced-based approach to improving the way that the hospital works. I have been trying to better understand the functioning of our outpatients department. Unfortunately, my suggestions for improving queuing, reducing waiting times, introducing triaging, and equitably distributing workload have been met with bureaucracy. Ironically, there is a fixation with 'statistics' in all the hospital departments - headcounts, bed occupancy, turnover - and an obsession with meeting 'targets'. However, no one has been able to explain how the numbers are derived, what the quality of the data is, what the resultant statistics actually mean, and how the religiously pursued targets were decided on. The true value of the information that can be generated is often lost in the pursuit of, and fixation on, end values. The impact of this tragedy is only realised when senior managers start basing important decisions, such as the need for more doctors, on these ill-obtained, often meaningless, numbers. I hope to make presentations on the basics of health measurement and information.

I have also been co-opted onto a project by the Benguela Health team who are working on improving the referral system in this district. They are piloting a multifunctioning referral letter, to improve communication between clinics and the hospital, bolstering of primary healthcare at clinics by promoting telephonic consultation with hospital-based doctors, promotion of closed loop communication and regular feedback, and including community health workers into the referral chain. The project speaks to the limitless potential to innovate in a resource-poor, low-technology setting.

\author{
Rubeshan Perumal \\ Nesri Padayatchi \\ Centre for the AIDS Programme of Research in South Africa (CAPRISA) \\ University of KwaZulu-Natal \\ Durban
}

Corresponding author: N Padayatchi (padayatchin@ukzn.ac.za) 